\title{
Recommendation Model Optimization Based on Diversity
}

\author{
Xiaofeng Li \\ Department of Information Science, \\ Heilongjiang International University, \\ Harbin 150025, China \\ mberse@126.com
}

\begin{abstract}
The accuracy of the traditional online recommendation system, much depends on the collaborative filtering recommendation algorithm, however, recommend system aims to attract the interest of consumers and turn visitors into buyers, rather than accurately predict their score. Online recommendation system is the service version of social filtering process. Most previous studies emphasize the accuracy of the collaborative filtering algorithm. However, the effective recommendation system must be credible. It requires that the system logic be transparency and the system be able to provide consumers a new, inexperienced item. Based on the above, this paper proposes to research the quality evaluation of recommendation system from the angle of user's experience, adding a freshness parameters of Top- $N$ recommend collaborative filtering similarity calculation method, and comparing with the classical recommended algorithm. The experiment result has a certain degree of accuracy and high diversity, which provides basis for establishing the e-commercial recommendation system.
\end{abstract}

Keywords: Recommend model, Diversity, Predictive scoring algorithm, Collaborative filtering recommendation algorithm

\section{Introduction}

With tremendous information provided by Internet, the efficiency and quality of information used by e-commerce system users are greatly declined because of information overload and disorientation. Recommendation systems can assist users in making choices from various alternative approaches [1-2]. The goal of such systems is to predict users' preference and provide prediction about appropriate information. Individualized recommendation technologies perform remarkably excellently in electronic commerce field. They can recommend according to consumers' interest and hobbies commodities which are consistent with their favors, substituting and surpassing the role of recommendation by traditional on-site stores' sales persons. The personalized suggestions are based on interaction or preferences, which thus offers suppliers a big opportunity $[3,4]$.

Ganesh [5] pointed out that online selling has become retailers' important strategy. After talking with online retailers, we found more than a half of them have planned or will plan to carry out recommendation function on their websites. Recommendation systems include artificial recommendation and computer recommendation, with the former including cross-selling finished by manpower [6-7]. The automatic recommendation system finished by computers is collaborative filtering, which utilizes data mining techniques to optimize interactive information with consumers for the purpose of personalized e-business. Automatic recommendation stresses real-time and machine learning algorithms rather than fixed data model [8-9]. The level of consumers' acceptance to recommendation systems has direct impacts on the effect of them. Being 
affected by many factors like personalization, source of recommended information and recommendation time, such systems play a dual role in consumers' decision-making process. To be specific, they can help consumers find really demanding products and have satisfied shopping experience and also stimulate product sales of merchants with effect on consumers' decision-making process. To solve those problems, we'll examine the recommendation mechanism based on service rating and consider the relationship between user preference similarity, recommendation trust and social relationship, as to improve the accuracy ratio of predicting and recommending product items by e-business systems [10].

\section{Recommendation Model Improvement based on Freshness Measurement}

Express rating data with a two dimensional scoring matrix. Let $U=\left\{u_{1}, u_{2}, \ldots u_{m}\right\}$ the collection of $\mathrm{m}$ users and the collection of $\mathrm{n}$ items is $I=\left\{i_{1}, i_{2} \ldots, i_{n}\right\}$. Users' rating data about one item is matrix $\mathrm{R}$ at $m \times n$ dimension. Every item $r_{u, i}$ in matrix $\mathrm{R}$ represents the score of item $\mathrm{i}$ by user $\mathrm{u}$. With evaluation matrix, we can compute the similarity between any two users and any two items. Then, we fetch from user's scoring files a certain number of training sets and test sets for the related experiment.

\subsection{KNN Measurement Method}

Among collaborative filtering recommendation algorithms, KNN's effect and efficiency influence those of collaborative filtering algorithms. Searching the nearest neighbor requires a measurement function which can measure similarities between samples. For different collaborative filtering recommendation methods, nearest neighbors are defined differently. For user-based algorithm, nearest neighbors are user group whose buying or scoring behavior is similar to the current users. The similarities between two users are obtained through some measuring method for dealing with the two user-item scorings.

2.1.1 User-based Similarity. Set user $\mathrm{x}$ and $\mathrm{y}$ which both have the itemset $I_{x, y}=I_{x} \cap I_{y}$ of rating data.

\section{User Similarity based on Pearson Measurement}

Pearson similarity is the commonest measuring method based on users. In the scoring matrix $\mathrm{R}$ of user against items, user x's rating for item $\mathrm{i}$ is $r_{x, i}$. The average score for user $\mathrm{x}$ and $\mathrm{y}$ is respectively $\bar{r}_{x}$ and $\bar{r}_{y}$. Then the similarity of user $\mathrm{x}$ and $\mathrm{y}$ based on Pearson similarity can be expressed as (1).

$$
\operatorname{sim}(x, y)=\frac{\sum_{i \in I_{x, y}}\left(r_{x, i}-\overline{r_{x}}\right)\left(r_{y, i}-\overline{r_{y}}\right)}{\sqrt{\sum_{i \in I_{x, y}}\left(r_{x, i}-\overline{r_{x}}\right)^{2}\left(r_{y, i}-\overline{r_{y}}\right)^{2}}}
$$

\section{User Similarity based on Cosine Method}

The scoring data is expressed with $\mathrm{n}$-dimensional vector. The vector value of those without evaluation value is 0 . User data is expressed with $\mathrm{m}$-dimensional vector. Through computation of cosine values between different angles, we can get user's similarity. The score of user $\mathrm{x}$ and $\mathrm{y}$ in $\mathrm{n}$-dimensional space is vector $\mathrm{x}$ and vector $\mathrm{y}$. Hence the relative similarity equation based on angle cosine method is shown in (2). 


$$
\operatorname{sim}(x, y)=\cos (\bar{x}, \bar{y})=\frac{\bar{x} \bullet \bar{y}}{\|\bar{x}\|_{2} *\|\bar{y}\|_{2}}
$$

Where, numerator equals to the inner product of two users' scoring vectors, while denominator is the product of two users' vector modules.

\section{User Similarity based on Amended Angle Cosine Method}

Instead of using traditional cosine methods which don't consider the problem with scoring magnitude of different users, lots of researchers applied the amended cosine method, based on which, the above problem is solved through deduction of mean scores. The user similarity based on the amended cosine method is like (3).

$$
\operatorname{sim}(x, y)=\frac{\sum_{i \in I_{x, y}}\left(r_{x, i}-\overline{r_{x}}\right)\left(r_{y, i}-\overline{r_{y}}\right)}{\sqrt{\sum_{i \in I_{x, y}}\left(r_{x, i}-\overline{r_{x}}\right)^{2} \sum_{i \in I_{x, y}}\left(r_{y, i}-\overline{r_{y}}\right)^{2}}}
$$

Set matrix $\mathrm{S}$ the similarity matrix between $n \times n$ dimensional users. Every $S_{x, y}$ in the matrix refers to the similarity between user $\mathrm{x}$ and $\mathrm{y}$. The above is the estimation of similarity generated based on users' historic rating records. If the number of products being definitely rated by a user is quite less, then the similarity got by that method is not accurate, as it's calculated based on the itemset which are appraised by both user $\mathrm{x}$ and $\mathrm{y}$. If the number of users increases, the computation amount grows linearly and performance gets worse.

2.1.2. Similarity Measurement based on iItems. Sarwar [11] et al. pointed out that user-based similarity has limitations in the face of massive data and dynamic update. They proposed to compute similarity of items based on collaborative filtering recommendation method. They had the similarity of both evaluated items and to-berecommended items. With similarity as weight to add scores of items which were already evaluated, they got the predicted scoring value of those not rated.

Item-based collaborative filtering method hypothesizes that: if most users have similar grades for one item, target users' scores for it may be the same. So it's possible to predict target users' rating against target item with calculation users' scoring records for similar items. The idea of item-based collaborative filtering is analogous to user-based collaborative filtering. The former focuses on item while the later concerns over user. Item-based calculation can be offline or completed before hand as per historical data, helpful to improve recommendation efficiency. The item-based similarity is acquired through:

\section{Item-based Pearson Similarity}

Define the collection $U$ of users who score both item $\mathrm{i}$ and $\mathrm{j}$. Their similarity is got by (4).

$$
\operatorname{sim}(i, j)=\frac{\sum_{u \in U}\left(r_{u, i}-\overline{r_{i}}\right)\left(r_{u, j}-\overline{r_{j}}\right)}{\sqrt{\sum_{u \in U}\left(r_{u, i}-\overline{r_{j}}\right)^{2} \sum_{u \in U}\left(r_{u, j}-\overline{r_{j}}\right)^{2}}}
$$

\section{Item-based osine Method}

Use vector $\bar{i}$ and $\bar{j}$ for user's evaluation of item. Their cosine similarity is reached by (5). 


$$
\operatorname{sim}(i, j)=\cos (\bar{i}, \bar{j})=\frac{\bar{i} \bullet \bar{j}}{\|\bar{i}\|_{2} *\|\bar{j}\|_{2}}
$$

\section{Item-based Amended Cosine Method for the Similarity}

The similarity between item $\mathrm{i}$ and $\mathrm{j}$ is obtained by amended cosine method like (6).

$$
\operatorname{sim}(i, j)=\frac{\sum_{u \in U}\left(r_{u, i}-\overline{r_{u}}\right)\left(r_{u, j}-\overline{r_{u}}\right)}{\sqrt{\sum_{u \in U}\left(r_{u, i}-\overline{r_{u}}\right)^{2} \sum_{u \in U}\left(r_{u, j}-\overline{r_{u}}\right)^{2}}}
$$

2.2 Predicted Rating Method. In synergic filtering system, the most important step is to produce output predicted ratings. With evaluation records of one item by other users, we can have user U's score $p_{u, i}$ for the product i. Set $\bar{u}$ the user collection of users who have rather high similarity. The function for predicting $p_{u, i}$ is:

$$
\begin{gathered}
p_{u, i}=\frac{1}{N} \sum_{u \in U} P_{\bar{u}, i} \\
p_{u, i}=K \sum_{u \in U} \operatorname{sim}(u, \bar{u}) P_{\bar{u}, i} \\
p_{u, i}=\bar{P}_{u}+k \sum_{u \in U} \operatorname{sim}(\overline{u, u})\left(P_{\bar{u}, i}-P_{u}\right)
\end{gathered}
$$

In which, $\operatorname{sim}(u, \bar{u})$ is similarity between user $\mathrm{u}$ and $\bar{u} ; \mathrm{k}$ is a standardized factor. $k=\sum_{u \in U} \mid \operatorname{sim}(u, \bar{u})$.The expression (7a) is used to directly compute the mean value of neighbors' scorings.

The weighted average algorithm equation (7b) is often used. The higher the similarity is between user $\mathrm{u}$ and $\bar{u}$, the weight of $P_{\bar{u}, i}$ is bigger. Thus, $(7 \mathrm{~b})$ can be used to measure the similarity in different recommendation systems.

Considering that (7b) uses simple and unified weighted value instead of estimating different assessing criteria by different users, the equation (7c) added based on personalized users differentials of mean values for varied degrees of preference, which has much higher accuracy ratio than the (7b).

The other technique for solving users' individualized rating problem is to utilize preference filtering. The strategy does not stress the absolute value of scores but predicts the relative preference value between different users.

Looking back to the long tail phenomenon implied by those recommendation evaluation information data after analysis, which contains a great deal of user demands, we develop the recommendation method based on diversity. By adjusting the process of it and sticking to maintenance of good precision ratio, we attempt to suggest long tail unpopular items which are potentially interesting to users to fulfill their experience and promote sales.

Based on the above analysis, the candidate recommendation item set added a fresh measure factor $\Phi_{\mathrm{F}}, \Phi_{\mathrm{F}} \in[0.1,0.9]$.This factor reflects the ratio recommended candidate set the long tail item total candidate recommendation item, The total number of hypothetical candidate recommendation item is 50 , which 40 is the popularity high item, 10 is popular degree low item, Proposed diversity measure collaborative filtering recommendation algorithm mainly steps are given in table 1 . 


\section{Experimental Analysis and Results}

We chose Movielens dataset from GroupLens Institute center of University of Minnesota. The rating data collection is applied to acquire watchers' scorings of movie items through the Internet, based on which, the algorithm predicts user preference as to select out the recommendation lists to send to users. We set 1-5 scores for different rating levels. The more scores mean audiences' high-degree satisfaction with the movie. Since implementation, the dataset has become one of the main datasets used by the institute centers about e-commerce recommendation methods.

\section{Table 1. Collaborative Filtering Recommendation Algorithm based on Diversity Measure}

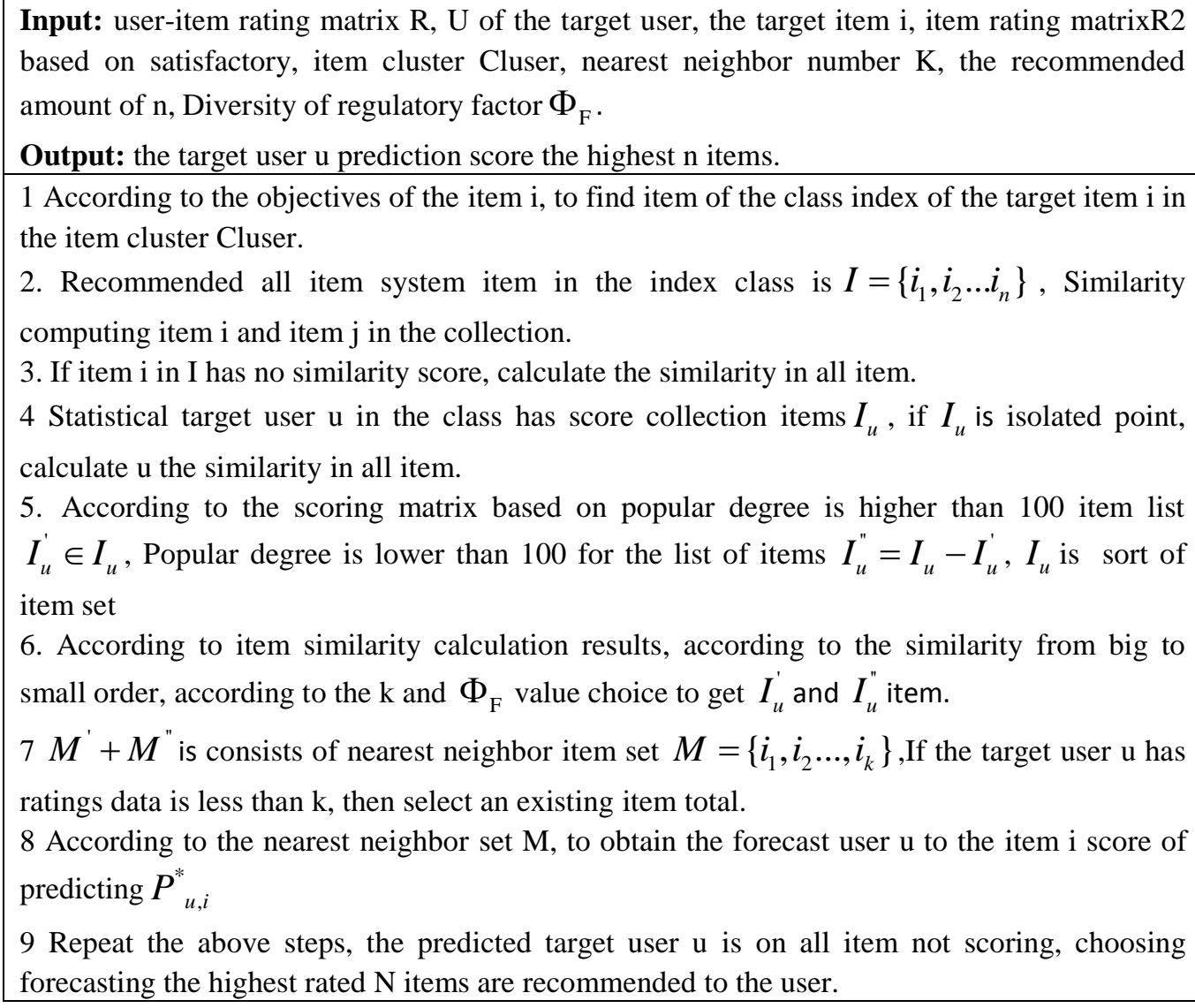

The test data used here are 100000 pcs of rating information chosen from the dataset Movielens, composed of 943 users' scorings against totally 1682 items. The experiment takes the usual five-fold cross validation: the dataset is crossed and divided to five subsets, with training set against test set at 4 versus 1 . Every time a subset is selected for the test. After consecutively five iterations, the mean value of all tests is adopted as the final result.

The experiment is made in Windows XP context. MATLAB used for algorithm coding.

\subsection{Classification of Movie Items in the Dataset}

According to features of movie items in the set, we classify movies into 19 types (Table 2). 
Based on item type, the item class matrix $\mathrm{G}$ is generated at $1683 \times 19$ dimension. When $\mathrm{i}$ item belongs to class $g_{i}, G_{i, j}=1$; or, $G_{i, j}=0$. Now we make clustering analysis for the item. Some items' class matrix is listed in Table 3.

For extreme sparseness of rating matrix, item class matrix needs to rely on simple categorization of item classes for higher clustering quality.

\subsection{Similarity Calculation Method}

For the clustering dataset, we need calculate the similarity between users and that between items. As for different datasets, the different results exist between item-based collaborative filtering similarity computation and user-based collaborative filtering similarity computation. We compared the effect of respectively user similarity method based on Pearson measuring, cosine similarity method and the modified cosine method. Learning that different similarity results could affect the accuracy of recommendation, we got the mean value of MAE after cross tests for 5 iterations for the chosen dataset. The results were presented in Fig. 1 and 2. Clearly, the modified cosine similarity method based on item performs the best in terms of recommendation accuracy. Therefore it is chosen for the comparison test in the next step. In the experiment, because it's required to pre-determine the value of initial clustering quantity Kcluster, the preset value of Kcluster has big influences on test results. From recommendation results got with different values of Kcluster, the recommendation accuracy is not consistent due to different $\mathrm{K}$ values. Here we chose Kcluster's best value $(\mathrm{K}=15)$ for the following control experiment.

Table 2. Movie Classification

\begin{tabular}{|l|l|}
\hline Item ID & Movie classification \\
\hline 0 & unknown \\
\hline 1 & Action \\
\hline 2 & Adventure \\
\hline 3 & Animation \\
\hline 4 & Children's \\
\hline 5 & Comedy \\
\hline 6 & Crime \\
\hline 7 & Documentary \\
\hline 8 & Drama \\
\hline 9 & Fantasy \\
\hline 10 & Film-Noir \\
\hline 11 & Horror \\
\hline 12 & Musical \\
\hline 13 & Mystery \\
\hline 14 & Romance \\
\hline 15 & Sci-Fi \\
\hline 16 & Thriller \\
\hline 17 & War \\
\hline 18 & Western \\
\hline
\end{tabular}

Table 3. Item Category Matrix Data Table

\begin{tabular}{|l|l|l|l|l|l|l|l|l|l|l|l|l|l|l|l|l|l|l|l|}
\hline $\begin{array}{l}\text { Item } \\
\text { ID }\end{array}$ & 0 & 1 & 2 & 3 & 4 & 5 & 6 & 7 & 8 & 9 & 10 & 11 & 12 & 13 & 14 & 15 & 16 & 17 & 18 \\
\hline 1 & 0 & 0 & 0 & 1 & 1 & 1 & 0 & 0 & 0 & 0 & 0 & 0 & 0 & 0 & 0 & 0 & 0 & 0 & 0 \\
\hline 2 & 0 & 0 & 0 & 0 & 0 & 0 & 0 & 0 & 0 & 0 & 0 & 0 & 0 & 0 & 0 & 0 & 1 & 0 & 0 \\
\hline
\end{tabular}




\begin{tabular}{|l|l|l|l|l|l|l|l|l|l|l|l|l|l|l|l|l|l|l|l|}
\hline 3 & 0 & 0 & 0 & 0 & 0 & 0 & 0 & 0 & 0 & 0 & 0 & 0 & 0 & 0 & 0 & 0 & 1 & 0 & 0 \\
\hline 4 & 0 & 1 & 0 & 0 & 0 & 1 & 0 & 0 & 1 & 0 & 0 & 0 & 0 & 0 & 0 & 0 & 0 & 0 & 0 \\
\hline 5 & 0 & 0 & 0 & 0 & 0 & 0 & 1 & 0 & 1 & 0 & 0 & 0 & 0 & 0 & 0 & 0 & 1 & 0 & 0 \\
\hline
\end{tabular}

\subsection{Method of Evaluating Statistical Accuracy}

3.3.1 Mean Absolute Error (MAE). The algorithm on the training set is the predicted ratings set $\left\{p_{1}, p_{2} \ldots, p_{n}\right\}$, the corresponding actual score in test is $\left\{q_{1}, q_{2} \ldots, q_{n}\right\}$, The value of MAE play score of recommendation system minus test set user on the target item practical value according to the training set to predict the target item scoring differential, by equation (8).

$$
M A E=\frac{\sum_{i=1}^{N}\left|p_{i}-q_{i}\right|}{N}
$$

Difference of predicted rating value and the actual value will reflect the quality of the user evaluation of recommender systems, the value of MAE index is small, it showed that the high prediction of recommended accuracy.

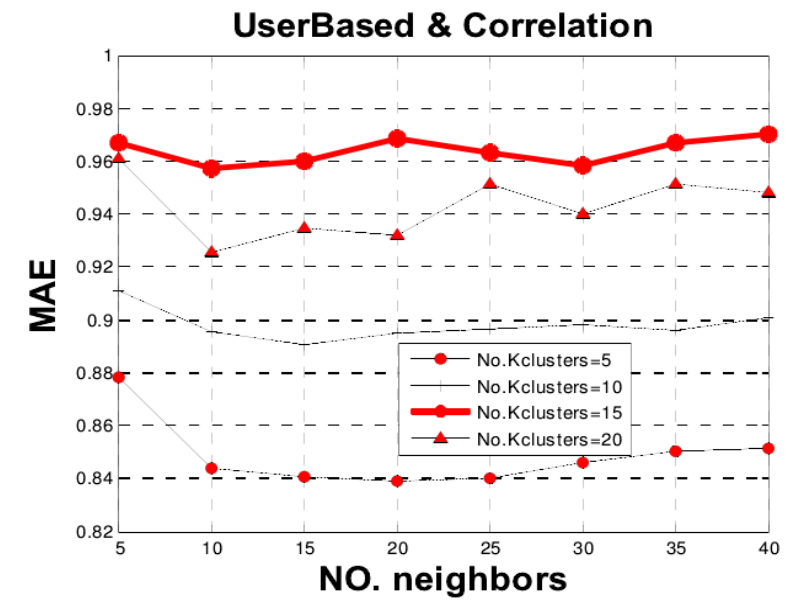

Figure 1. MAE of User Similarity based on Collaborative Filtering

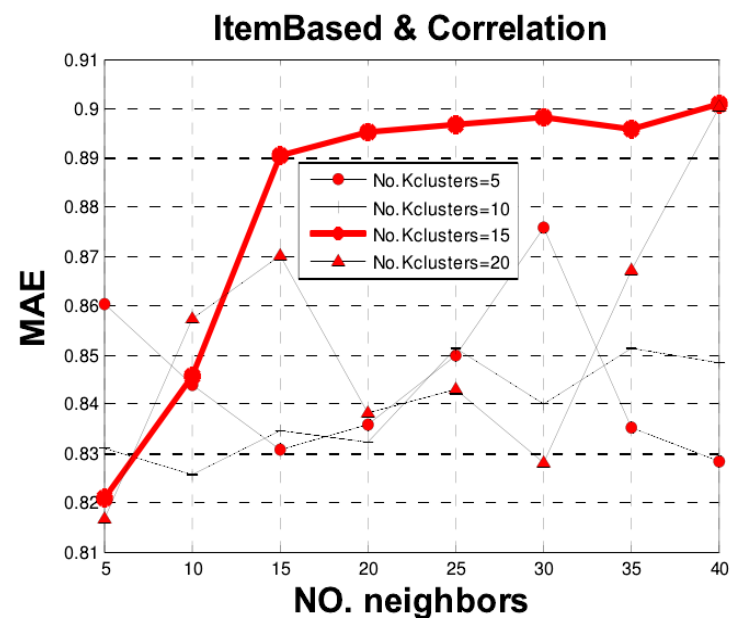

Figure 2. MAE of Item based on Collaborative Filtering based on 
3.3.2 Precision. TOP-N recommended result set of user $u$ is $T$, the accuracy of prediction is by formula (9).

$$
\text { Precision_N } N=\frac{1}{|U|} \sum_{u} \frac{\left|T_{u} \cap R\right|}{N}
$$

3.3.3 Coverage. Prediction coverage is by formula (10), diversity is the overall evaluation.

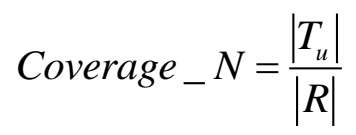

3.3.4 Diversity. Diversity prediction result is by formula (11).

$$
D I V_{-} N=\frac{1}{|U|} \sum_{u}(1-\operatorname{sim}(R, u)) / 2
$$

\subsection{The Contrast Experiment}

To increase the diversity factor, based on item similarity and user similarity were calculated, comparison of recommended results is shown in Figure 3 and figure 4.

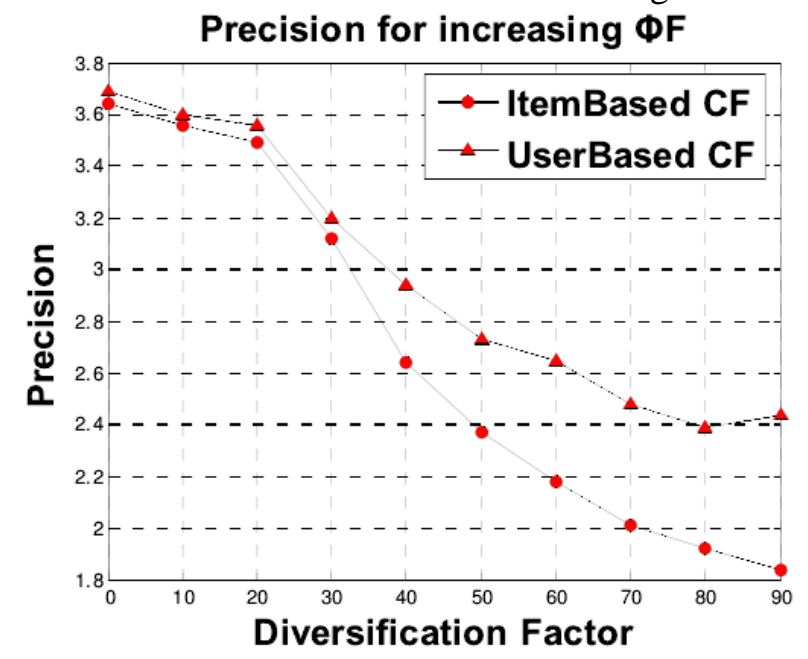

Figure 3 Precision Measure based on Diversity

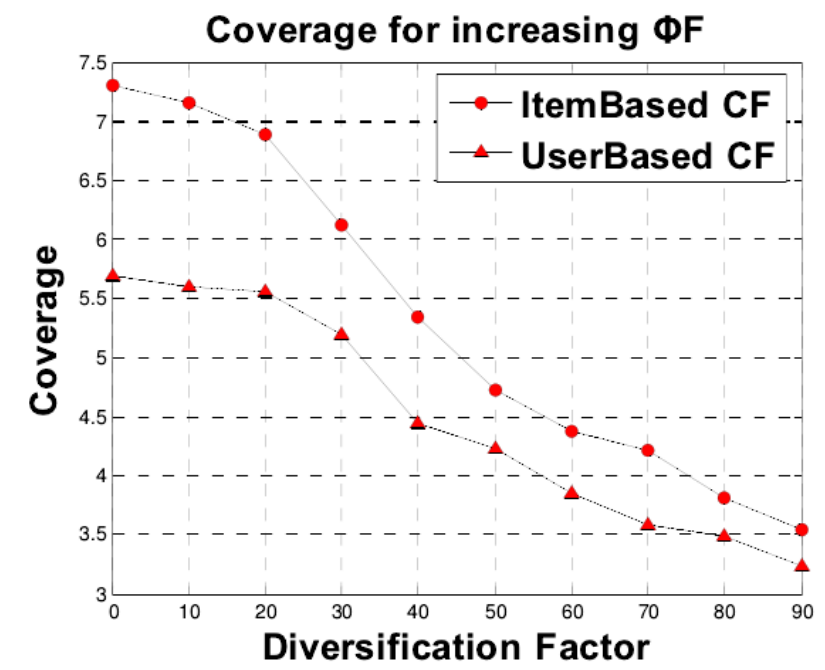

Figure 4. Coverage Measure based on the Diversity 
In this section, it hopes to validate the effect of item clustering on the result. The control test is to confirm the active role of diversity in the collaborative filtering.

The control experiment methods include:

1) $\mathrm{N}$ random recommendation method (Random-N);

2) Standard recommendation, i.e. Top-N items with higher scores (TraditionCF);

3) Pop recommendation, i.e. Top- $\mathrm{N}$ items which are ranked completely as per the popularity (POP-N);

4) diversity-based collaborative filtering method (DiversityCF)

MAE values, accuracy ratio, coverage and diversity of the four techniques are seen in Fig. 5 and 8. It's obvious from them that:

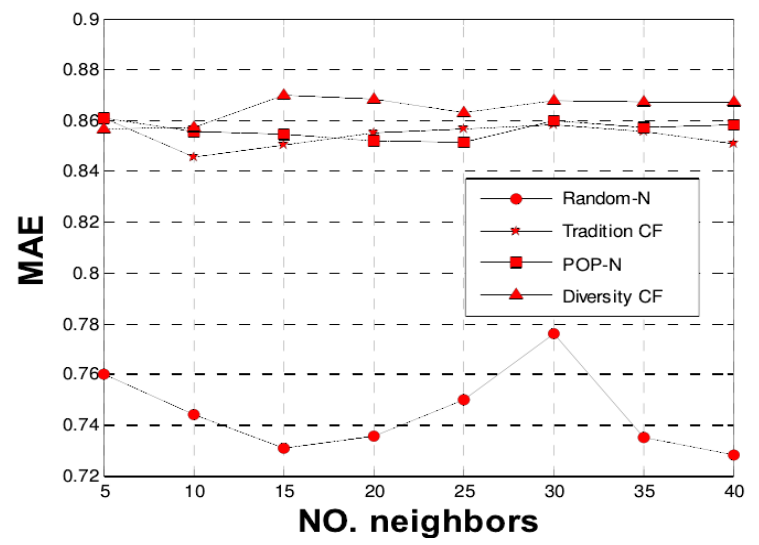

Figure 5. Comparison of Experimental Results based on MAE

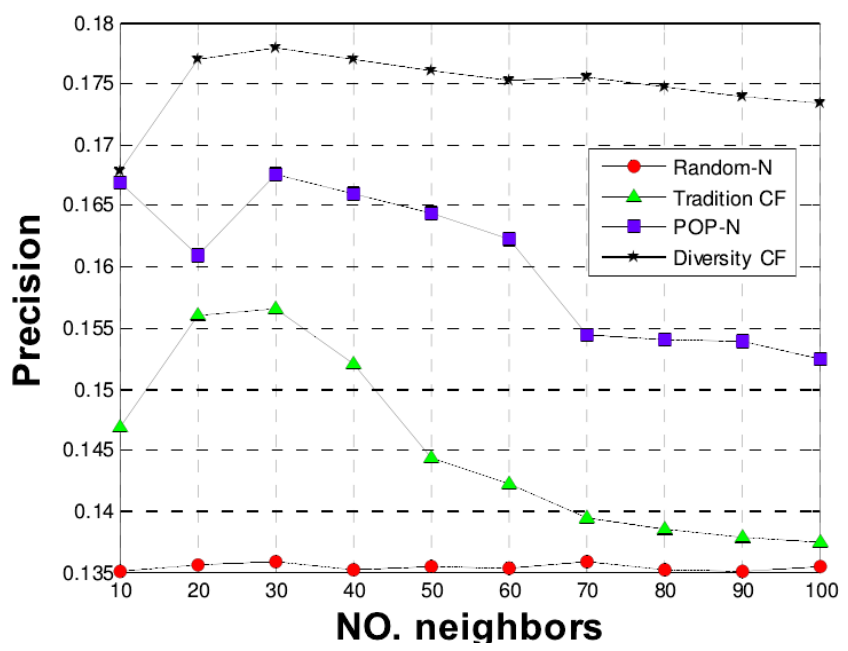

Figure 6. Comparison of Experimental Results based on Precision 


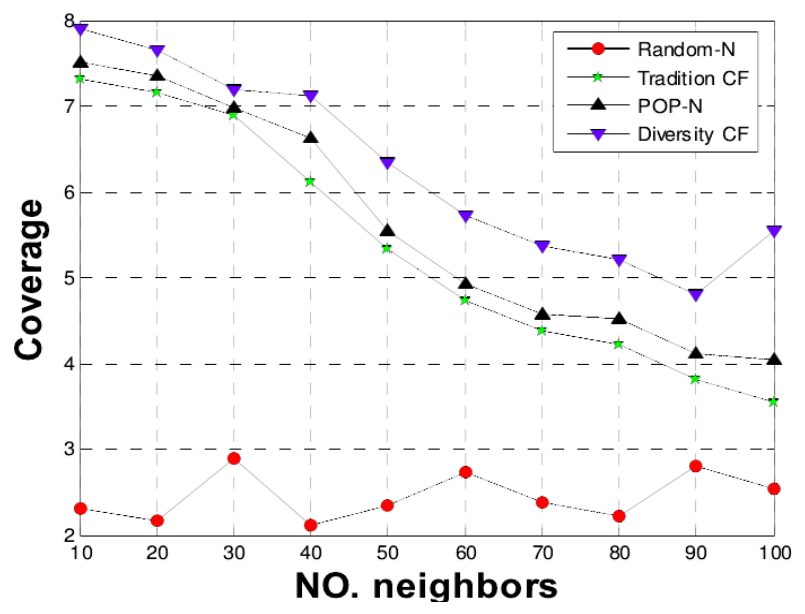

Figure 7. Comparative Experiment Results based on Coverage

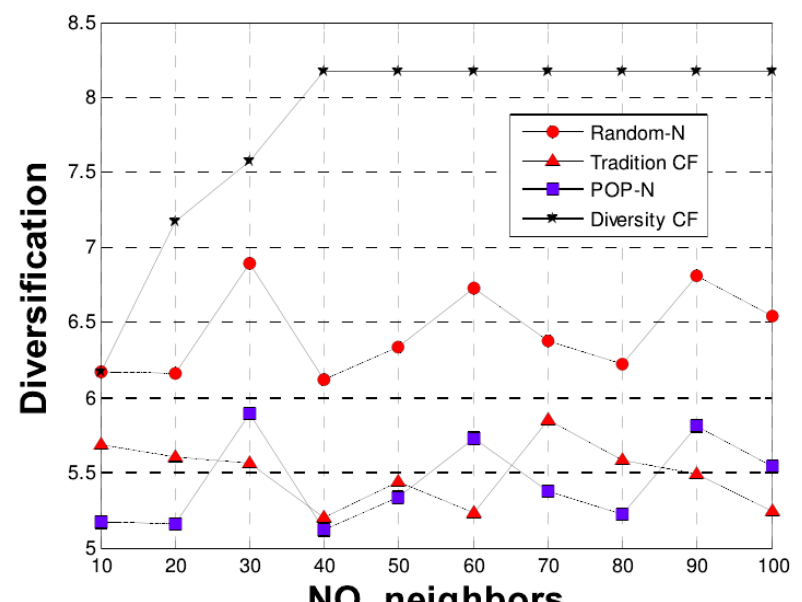

Figure 8. Comparison Experimental Results based on Diversity

Diversity is enhanced at the cost of scarifying certain recommendation accuracy rate. When observing the recommendation result with different values of $\Phi_{\mathrm{F}}$, we found that MAE and the accuracy declined along with the increase of $\Phi_{\mathrm{F}}$. Among them, user-based collaborative filtering method and item-based method have slightly stable MAE and the accuracy values when $\Phi_{\mathrm{F}}$ changes in $[0,0.2]$, but drop rapidly after $\Phi_{\mathrm{F}}=0.3$. To improve recommendation result diversity in the meantime of maintaining certain accuracy, we chose $\Phi_{\mathrm{F}}=0.2$ for further test.

We selected random recommendation method, traditional collaborative filtering recommendation method and popularity recommendation method to compare with the method for reinforcing diversity measurement. The experiment result shows that the random method has the lowest accuracy rate and coverage. The number of candidate neighbors affected little the recommendation result. The traditional standard collaborative filtering and diversity-based recommendation method both have better precision rate than the other two. The diversity-based method improved the coverage rate and diversity of recommendation results while maintaining recommendation precision. 


\section{Conclusion}

It outlined the progress that collaborative filtering recommendation method made from the mere method to user experience. Then it proposed the recommendation model based on variety. The recommendation quality was enhanced by adjusting the percentage of long-tail items through augmentation of freshness parameter when the candidate recommendation item set was calculated. The simulation experiment was designed to evaluate the diversity-based recommendation algorithm. The nearest neighbor quantity, clustering quantity and parameter value were appropriately selected. The result confirmed that the proposed method is superior in diversity and coverage rate while keeping certain accuracy rate.

\section{Acknowledgements}

This work is supported by The Education Department of Heilongjiang province science and technology research project, Under Grant No. 12543076

\section{References}

[1] W. H. Li and S. Xu, "The design and implementation of e-commerce recommendation system based on Hadoop", Computer engineering and design, no. 1, (2014), pp. 130-136.

[2] B. B. Wang, Z. J. J. Zhou and J. G. Pan, "Iterative training Web Service hybrid collaborative filtering recommendation model based on computer", Research and development, no. 2, (2013), pp. 153-162.

[3] J. M. Hu and A. Mandi, "On the socialization of minor user resource association recommendation model based", Information theory and practice, no. 4, (2014), pp. 123-126.

[4] Z. S. Wang, Q. Li and W. J. Su, "Large scale personalized recommendation based on implicit feedback", Journal of software, no. 9, (2014), pp. 1953-1966.

[5] J. Ganesh, K. E. Reynolds and M. Luckett, "Online shopper motivations, and e-store attributes: an examination of online patronage behavior and shopper typologies", Journal of Retailing, vol. 86, no. 1, (2010), pp. 106-115.

[6] X. G. Yang, T. I. Jiong, Q. Yurong and S. Hua, "Consider the collaborative filtering recommendation model item property", Computer applications, vol. 11, (2013), pp. 3062-3066.

[7] C. H. Ju and F. G. Bao, "The situation and the main features of multi dimension of research into personalized recommendation model based", Journal of communication, no. 1, (2012), pp. 17-27.

[8] Y. G. Guo and K. B. Chen, "Recommendation model current news concerned products", Computer applications and software, vol. 10, (2009), pp. 162-165.

[9] L. H. Ji Lianghao, "Research on Collaborative Filtering Recommendation Based on user information", Computer engineering and design, no. 8, (2008), pp. 2047-2051.

[10] J. Y. Sun and X. L. Yu, "Recommendation model oriented semantic search", Journal of Guangxi Normal University (Natural Science Edition), no. 3, (2008), pp. 202-205.

[11] B. Sarwar, G. Karypis, J. Konstan and J. Riedl, "Item-based collaborative filtering recommendation algorithms", Proc 10th Int. Conf., (2001); Hong Kong.

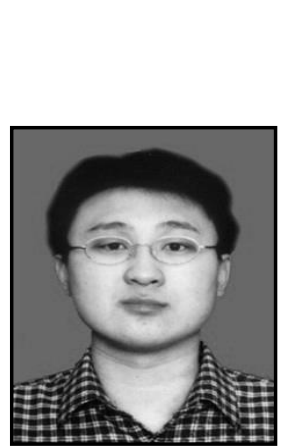

\section{Author}

Li Xiaofeng, he is an advanced member of china computer federation and he is an associate professor in Heilongjiang International University. His research interest includes data mining and intelligent algorithm. 
International Journal of $u-$ and e- Service, Science and Technology Vol.8, No.5 (2015) 